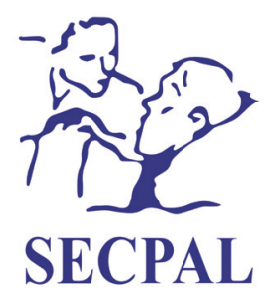

Medicina Paliativa

www.medicinapaliativa.es

ORIGINAL

\title{
El pacto de silencio en el final de la vida. Un análisis cualitativo sobre la perspectiva de los implicados en el fenómeno
}

\author{
Cristina Lope Mateo ${ }^{1}$ y José Luis Díaz Agea*2
}

${ }^{1}$ Equipo de Cuidados Paliativos Domiciliarios (ECPD), Área IX, Murcia, España. ${ }^{2}$ Facultad de Enfermería, Universidad Católica de Murcia, España.

Recibido el 12 de febrero de 2018

Aceptado el 9 de mayo de 2018

\author{
PALABRAS CLAVE \\ Información médica, \\ comunicación en \\ salud, conspiración \\ del silencio, paciente \\ terminal, \\ investigación \\ cualitativa.
}

\begin{abstract}
Resumen
Introducción: El pacto de silencio, a pesar de ser una situación relativamente frecuente, ha sido poco explorado desde el punto de vista de los sujetos que lo protagonizan.

Objetivo: Analizar y profundizar en la comprensión del fenómeno del pacto de silencio en el final de la vida a través de las perspectivas de todos los implicados (paciente, familiares y profesionales).

Método: Estudio exploratorio con metodología cualitativa realizado en el Área IX del SMS de la Región de Murcia, mediante la técnica del grupo focal (2 grupos focales de profesionales y 1 grupo focal de familiares de enfermos terminales), así como el método biográfico (mediante la narrativa/historia de vida de una paciente en fase terminal).

Resultados: La conspiración del silencio repercute en el afrontamiento de la enfermedad terminal. Existe un gran componente emocional y cultural que bloquea en ocasiones la comunicación entre profesionales, familia y paciente. Los profesionales de cuidados paliativos afrontan mejor la relación y la comunicación con el paciente terminal que los de atención primaria. El paciente consideró que el conocimiento de su diagnóstico/pronóstico fue beneficioso.

Conclusiones: El pacto de silencio tiene un marcado carácter sociocultural. No se puede responsabilizar a los profesionales o a los familiares de la situación sin antes considerar el contexto en el que se desarrolla (muerte como tabú moderno). Es preciso que los profesionales que atienden a pacientes terminales se formen en cuidados paliativos y en habilidades de comunicación.
\end{abstract}

*Autor para correspondencia:

José Luis Díaz Agea

Universidad Católica de Murcia. Avda Jerónimos S/N. 30107, Guadalupe, Murcia

Correo electrónico: jluis@ucam.edu 


\section{KEYWORDS}

Medical information, health communication, conspiracy of silence, terminal patient, qualitative research.

\begin{abstract}
Introduction: The pact of silence, despite being a relatively frequent situation, has been hardly explored from the point of view of the subjects involved.
\end{abstract}

Objective: To analyze and understand the characteristics of the phenomenon through the perspectives of all those involved (patient, family, and professionals).

Method: A study with qualitative methodology carried out in Area IX of the SMS (Murcia region health system) using a focal group approach (2 groups of professionals and 1 group of relatives of the terminally ill), as well as a biographical method (using the narrative/life history of the terminal patient).

Results: The conspiracy of silence negatively impacts coping in the terminally ill. There is a large emotional and cultural component that sometimes blocks communication between professionals, family, and patients. Palliative care professionals are better suited than primary care providers to deal with relationships and communications with terminal patients. Patients considered that knowledge of their diagnosis/prognosis was beneficial.

Conclusions: The pact of silence has a marked sociocultural character. Professionals or relatives cannot be held responsible for this situation without considering the context in which it takes place (death as a modern taboo). Professionals who care for terminal patients must be trained in palliative care and communication skills.

Lope Mateo C, Díaz Agea JL. El pacto de silencio en el final de la vida. Un análisis cualitativo sobre la perspectiva de los implicados en el fenómeno. Med Paliat. 2019;26(2):127-135.

\section{Introducción}

El pacto de silencio se produce cuando se acuerda (implícita o explícitamente), por parte de los profesionales y seres queridos ${ }^{1,2}$, alterar $u$ omitir la información transmitida al enfermo ${ }^{3}$ relativa al diagnóstico y/o pronóstico sobre la gravedad de la enfermedad, de forma parcial o total, con el objetivo de protegerle del sufrimiento que pueda experimentar ${ }^{4}$ en un contexto habitualmente de terminalidad. Hay que resaltar que, en nuestro marco legal ${ }^{1}$, la autonomía del paciente se ha reconocido como un derecho fundamental, en virtud del cual el paciente es el titular del derecho a la información sobre su enfermedad. En cuanto a los términos "pacto de silencio" y "conspiración del silencio", en la literatura se usan indistintamente, pero en la actualidad ${ }^{5}$ se prefiere "pacto de silencio", dado el carácter negativo del término "conspiración". Es por ello que, a lo largo de este artículo, se usará preferentemente el término "pacto".

A pesar de que el paciente es el titular del derecho a la información, en la práctica, los cuidadores familiares son los principales destinatarios de la información en situaciones de riesgo vital para el ser querido 6 . Los principales motivos para ocultar la información al paciente suelen ser producto de una reacción inicial de shock, fruto de la ansiedad y del temor a la muerte ${ }^{7}$, así como la intención benevolente ${ }^{1}$ basada en la evitación de un daño añadido al sujeto por su reacción al conocer detalles de la enfermedad que padece. También se han descrito motivos relacionados con las dificultades de comunicación de los profesionales al respecto de dar malas noticias, o la creencia en el empeoramiento del paciente al conocer un pronóstico desalentador ${ }^{5}$.
En la literatura aparecen argumentos en contra de esta práctica, basados en las consecuencias negativas que tiene, sobre todo, para los pacientes (no elaboración del duelo, falta de comunicación y menor expresión de deseos o miedos que les llevaría a una situación de aislamiento). Asimismo, se han descrito diferentes comportamientos desadaptativos familiares según el grado de información que se le brinda al paciente ${ }^{8}$. Esto va a influir en el funcionamiento familiar y en la atención al mismo ${ }^{9}$. Para los profesionales, la situación relacionada con la comunicación de un pronóstico desalentador no está exenta de impacto emocional ${ }^{10,11}$, aunque las recomendaciones sobre una adecuada práctica profesional vienen de la mano de considerar cada caso particular y valorar la necesidad de información de cada paciente, actuando en consecuencia, de la manera más ética posible y con unas adecuadas habilidades de comunicación ${ }^{5}$.

Sin embargo, se han descrito ${ }^{12,13}$ algunos aspectos positivos de esta conducta de ocultación de la verdad al paciente, como la evitación de reacciones emocionales imprevisibles ante los profesionales por parte de la familia o el enfermo, o también se ha argumentado cómo la normalidad aparente facilitaría cierta tranquilidad en un contexto en el que culturalmente se acepta de forma tácita el mantener al paciente en la ignorancia de su enfermedad para protegerlo (cuestión en la que la cultura anglosajona es más directa que la mediterránea).

El pacto de silencio, a pesar de su frecuencia, ha sido poco explorado desde el punto de vista de sus protagonistas, precisándose estudios que analicen con profundidad las causas y factores que intervienen en el mismo ${ }^{14}$. El objetivo de esta investigación ha sido analizar y profundizar en la comprensión del fenómeno del pacto de silencio en el final de la vida a través de las perspectivas de todos los implicados 
(paciente, familiares y profesionales). El propósito último será la transferencia de los resultados a la práctica clínica para atender de un modo más eficiente a las necesidades de los pacientes, familiares y profesionales implicados en el cuidado de las personas en el contexto del final de la vida desde una perspectiva más humanística ${ }^{15}$.

\section{Material y métodos}

Estudio exploratorio, descriptivo con metodología cualitativa ${ }^{16}$. La recogida de datos se ha llevado a cabo mediante la técnica del grupo focal ( 2 grupos focales de profesionales y 1 grupo focal de familiares de enfermos terminales), así como el método biográfico ${ }^{17}$ (una entrevista biográfica de una paciente informada del diagnóstico y pronóstico de su enfermedad en fase terminal). La investigación se ha realizado durante el periodo comprendido entre marzo y septiembre de 2017 en el Área de Trabajo del Equipo de CP Domiciliario del Área IX del SMS de la Región de Murcia, que comprende el Área IX Vega Alta del Segura y Hospital Morales Meseguer.

La selección de la muestra se realizó con criterios no probabilísticos y de conveniencia, y teniendo en cuenta los criterios inclusión/exclusión (Tabla I). En lo que respecta a los grupos focales se siguieron criterios de homogeneidad/ heterogeneidad como recomienda la literatura ${ }^{18}$. Se escogieron como informantes a profesionales sanitarios ( 2 médicos, 3 enfermeros y 1 trabajadora social) que trabajan en Atención Primaria (AP), constituyendo el grupo focal 1 (GFP1), así como profesionales sanitarios del Hospital Morales Meseguer (centro de referencia oncológica del área) (3 médicos, 2 enfermeros, 1 trabajadora social), formando el grupo focal 2 (GFP2). Para la entrevista biográfica (paciente terminal) se seleccionó a una única paciente incluida en $\mathrm{CP}$.

Para el grupo focal de familiares (GFF) se escogieron a 5 familiares de pacientes.

Se recabó información de aspectos referidos a las siguientes dimensiones (Tabla II).

Se recogió información creando un clima de confianza entre los participantes para así favorecer la exposición de sus opiniones y experiencias. La duración de cada grupo focal fue de aproximadamente una hora. Previamente, se elaboró un guion (Tabla III) para los grupos focales de profesionales y otro para el grupo focal de familiares. Para la historia de vida se tuvieron varios encuentros con la paciente y se realizó en forma de varias entrevistas biográficas con un guion semiestructurado.

El análisis se realizó una vez transcritos los archivos de audio y las notas registradas en el cuaderno de campo, utilizando un programa de codificación y análisis cualitativo (MaXQDA v. $10^{\circ}$ ). Para asegurar la fiabilidad de los datos, como es propio de la investigación cualitativa, se usó la estrategia de reflexividad (todos los miembros del equipo de investigación consideraron sus propias suposiciones internas y externas sobre el fenómeno de estudio a la hora de recopilar y analizar los datos). Para evitar el sesgo de parcialidad (la interacción del entrevistador puede influir en las respuestas del entrevistado), se evitó en todo momento manifestar las propias opiniones durante el desarrollo de los grupos focales o la entrevista biográfica. No obstante, hay que señalar que la naturaleza interactiva de la investigación cualitativa es en sí misma una fortaleza y resultó incluso terapéutico para profesionales, familiares y paciente, al poder sacar emociones e ideas que nunca habían compartido con otras personas.

También se usó la triangulación de instrumentos, datos e investigadores (la trascripción y la interpretación de los resultados fueron corroborados por dos investigadores de manera independiente).

El discurso se sometió a análisis de contenido sistemático y basado en categorías a priori establecidas (dirigido) ${ }^{19}$ para su codificación. Asimismo, para la interpretación de los datos se utilizó el contrastado método de Colaizzi ${ }^{20}$, recomendado para la investigación social. Dicho estilo de análisis consta básicamente de los siguientes pasos:

1. Transcripción literal de las entrevistas. Lectura varias veces de la descripción del fenómeno de cada participante.

2. Volver a la transcripción original y releer para extraer las declaraciones significativas.

3. Identificar unidades de significados de cada una de las declaraciones significativas.

4. Organizar las unidades de significado, agruparlas en categorías comprensivas mayores (definidas a priori como temas generales a tratar en nuestro caso).

5. Escribir una exhaustiva descripción de fenómeno en estudio, describiendo las categorías y unidades de significado construidas.

6. Devolver a los participantes para validar la descripción.

7. Si se revelan nuevos datos durante la validación, se deben incorporar dentro de la descripción exhaustiva.

Se siguieron los criterios éticos relacionados con la investigación cualitativa (consentimiento informado, confiden-

Tabla I. Criterios de inclusión y exclusión en el estudio

$$
\text { Criterios de inclusión }
$$

Profesionales: pertenecer a un equipo de AP u Hospital que trabaje con pacientes en situación terminal del Área IX del SMS de la Región de Murcia

Familiares: ser familiares de pacientes que han sido atendidos en el Programa de CP Domiciliario del Área IX del SMS de la Región de Murcia

Paciente: estar en situación terminal y conocer su pronóstico. Estar incluido en el Programa de CP Domiciliario del Área IX del SMS de la Región de Murcia

\section{Criterios de exclusión}

Profesionales: no estar en activo o llevar más de 2 años sin ejercer su labor con pacientes terminales Familiares: encontrarse en proceso de duelo patológico o bajo tratamiento psiquiátrico

Paciente: no estar en plenas facultades mentales o en una condición física o psíquica que le impida ser entrevistado. Encontrarse en una etapa del duelo diferente a la aceptación plena de su situación 
Tabla II. Dimensiones del estudio

\begin{tabular}{ll}
\hline \multicolumn{1}{c}{ Dimensión } & \multicolumn{1}{c}{ Descripción } \\
\hline Percepción del proceso salud/enfermedad/atención & $\begin{array}{l}\text { Cómo responde el paciente ante la información del proceso } \\
\text { de pérdida grave de la salud }\end{array}$ \\
\hline Manejo de la información & $\begin{array}{l}\text { Cómo, cuándo y por qué se maneja la información en el } \\
\text { contexto de la terminalidad }\end{array}$ \\
\hline Identificación de las dificultades de relación y comunicación & Con paciente terminal y familia en la fase final de la vida \\
\hline Abordaje del enfermo terminal & $\begin{array}{l}\text { Conocer la opinión de los profesionales sobre cómo se } \\
\text { sienten durante la atención del enfermo en su terminalidad }\end{array}$ \\
\hline Estrategias de afrontamiento de la enfermedad & En función de la información recibida \\
\hline \multirow{2}{*}{ Relación según el entorno } & $\begin{array}{l}\text { Opinión de los participantes sobre cómo afectan diversos } \\
\text { factores como las creencias religiosas, el nivel sociocultural y } \\
\text { la sociedad en el proceso de la enfermedad }\end{array}$ \\
\hline
\end{tabular}

Tabla III. Guion de temas a tratar/preguntas en grupos focales y entrevista biográfica

\begin{tabular}{|c|}
\hline Guion grupo focal profesionales \\
\hline $\begin{array}{l}\text { Describir una experiencia en la que se haya producido conspiración del silencio } \\
\text { ¿Por qué crees que se produce la conspiración del silencio? } \\
\text { ¿Cuál es tu opinión sobre el aportar o no información del diagnóstico y pronóstico a un paciente en fase terminal? } \\
\text { ¿Qué es lo que ocurre una vez explicado el diagnóstico y pronóstico? } \\
\text { ¿Crees que es mejor morir sin tener conocimiento del proceso de la enfermedad? } \\
\text { ¿Cómo te sientes hablando de la enfermedad terminal con el paciente y/o la familia? } \\
\text { ¿Cómo crees que influye al paciente el tener conocimiento de la información sobre su gravedad? } \\
\text { ¿Cómo influyen las creencias religiosas en el afrontamiento de la enfermedad? } \\
\text { ¿Cómo se ve la muerte en la sociedad actual? } \\
\text { ¿Aspectos como el nivel sociocultural pueden influir en el cuidado del paciente por parte de la familia? }\end{array}$ \\
\hline Guion grupo focal familiares \\
\hline $\begin{array}{l}\text { Breve presentación de su experiencia personal } \\
\text { ¿Qué opinas sobre informar al ser querido sobre su gravedad? } \\
\text { ¿Crees que es mejor morir sin tener conocimiento de la enfermedad? } \\
\text { ¿Qué crees que puede pasar si se informa al ser querido sobre su diagnóstico y pronóstico? } \\
\text { ¿De qué manera ha repercutido en vosotros emocionalmente el proceso de la enfermedad de vuestro ser querido? } \\
\text { ¿Ha influido en vosotros la creencia religiosa? }\end{array}$ \\
\hline Método biográfico (guion semiestructurado) paciente \\
\hline $\begin{array}{l}\text { Varios encuentros en los que se habla sobre el antes y el después de contraer la enfermedad } \\
\text { Información sobre la enfermedad } \\
\text { Cómo es la situación actual } \\
\text { Principales preocupaciones en el momento actual } \\
\text { Expresión de sentimientos sobre el pasado y el presente, tomando como hito la enfermedad } \\
\text { ¿Cómo crees que está tu familia? } \\
\text { ¿Habláis de lo que sientes? } \\
\text { Expresión de las creencias y del marco de sentido } \\
\text { ¿Hubieras preferido no saber la gravedad de tu enfermedad? ¿Por qué? }\end{array}$ \\
\hline
\end{tabular}

cialidad y manejo de riesgos). Se obtuvo la aprobación del Comité de Ética de la UCAM para la realización del proyecto (n. ${ }^{\circ}$ Ref: 6660/2017). La información fue transcrita evitando elementos informativos sobre los participantes, asignando a cada entrevistado un código de identificación, preservando así su anonimato. El sistema de códigos asignado fue GFP1/ GFP2 (grupo focal de profesionales 1 y 2 ), seguido de $M 1, M 2$ (médico 1, médico 2...) ENF1, ENF2 (enfermera 1, etc.); GFF (Grupo focal de familiares) seguido de F1, F2, etc. (Familiar 1,2 , etc.) y para las opiniones del paciente se usó PAC1.

\section{Resultados}

\section{Reacción sobre la información de la enfermedad}

Para los profesionales, en el momento de recibir la información el paciente suele experimentar un impacto emocional. Esta reacción es común también en la familia. Posteriormente, suelen sucederse distintas fases (coincidiendo con las descritas en los estudios de duelo) hasta alcanzar, 
en ocasiones, un periodo de calma y de agradecimiento que facilita el poder solucionar cuestiones pendientes.

"Teniendo en cuenta que cada situación es completamente única, así de manera general, hay una tormenta emocional y después viene la calma y en la mayoría de las situaciones todo es más fácil..." GFP1 (MED2).

Algunos cuidadores afirman que sus seres queridos no recibieron información sobre el diagnóstico. Ellos, al recibir la noticia, sufrieron un gran impacto emocional y creen que si su familiar lo hubiera sabido experimentaría tristeza, pero tras un tiempo lo hubieran superado. También creen que la mayoría de sus familiares intuían o sabían su pronóstico, y el hecho de informarles no habría empeorado el transcurso de la enfermedad. El paciente, sin embargo, expresaba de este modo cómo experimentó el momento en que supo su pronóstico:

“...Mal, me hundí y no paraba de llorar. No me creía lo que tenía y no tenía ganas de nada, solo de llorar..."

\section{Manejo de la información}

Se exploró el conocimiento sobre la enfermedad y la información. Los profesionales creen que la mayoría de los pacientes son conscientes de la gravedad de su situación. Creen que hay que adaptar la información a cada situación particular. En muchos casos se propicia que sea el paciente quien la demande. Una idea generalizada en los profesionales es nunca mentir al paciente.

“Hay gente que prefiere morir en la ignorancia” GFP1 (ENF2).

"Creo que al final todos se enteran porque se les haya dicho o porque no (...)" GFP2 (ENF2).

Los familiares opinan que la información que se da al paciente depende de factores personales (momento vital en el que aparece la patología y edad del paciente).

“(...) él en cierto modo no quería saber totalmente la verdad, porque a él le hacía daño. Yo intenté hablar en un par de ocasiones con él y se hundía (...)" GDF (FAM1).

"Mi esposo se encontraba mal y él mismo quiso saberlo, bueno, no al principio, pero conforme iba pasando (...) sí lo sabía (...) pero él no quería preguntar a los doctores, se lo recelaba..." GDF (FAM3).

En el caso del paciente fue importante conocer con claridad el diagnóstico y las posibilidades terapéuticas, así como su evolución y desenlace.

"Quise saberlo desde el principio y siempre he querido estar informada (...) quiero luchar todo lo posible hasta el final" PAC1.

\section{Identificación de las dificultades de relación y comunicación}

Se exploraron los motivos que conducen a no comunicar al paciente la gravedad de su situación. La mayoría de los profesionales creen que es debido a los propios miedos ("miedo" es la palabra que más se repite en el análisis de contenido), por tener que informar al paciente sobre algo que no quiere conocer o por miedo a la reacción del paciente.

Se habla de una base cultural del problema (la costumbre en España determinaría que la información médica se proporcione primero a los familiares, y es la familia la que toma la decisión de informar o no al ser querido). También aparece la edad avanzada como una causa que influye en que se oculte la información.

“(...) hemos cambiado poco cuando es el paciente el que tiene que recibir la información y sin embargo seguimos informando a la familia (...) porque la cultura está ahí y no la podemos hacer desaparecer de un día para otro" GFP1 (MED 1).

Los familiares creen que términos como "cáncer" y "muerte" se consideran un tabú social.

También aparece la edad como determinante para ocultar información.

"Tú vas a cualquier centro médico, tú hablas de cualquier enfermedad, los ves en los centros médicos hablando, pero la palabra 'cáncer' nadie la nombra” GFF (FAM5).

También es necesario destacar que la protección del estado de ánimo del paciente y el respeto por aquellos que no desean saber (motivo compasivo) fue uno de los argumentos expresados por una participante al comentar este aspecto en el grupo de familiares.

"Depende del caso, el estado del enfermo... Yo en el caso de mi marido, él en cierto modo no quería saber totalmente la verdad, porque a él le hacía daño. Yo intenté hablar en un par de ocasiones con él y se hundía..." GFF (FAM1).

Esta dimensión no se analizó en PAC1, dado que conocía toda la información sobre la enfermedad y su gravedad.

\section{Abordaje del enfermo terminal}

Se exploró cómo se sienten los participantes durante la atención en el final de la vida (Figura 1). Cabe destacar la diferencia de opinión del grupo de profesionales de AP con respecto a los de CP. En general, los profesionales de AP se sienten tensos y les incomoda tener que atender a un paciente terminal.

"Yo me encuentro tenso y alerta y midiendo las palabras. No estás hablando de forma distendida..." GFP1 (MED2).

"La verdad es que te carga mucho y terminas agotada porque te transmite tristeza" (GFP1) TS. 


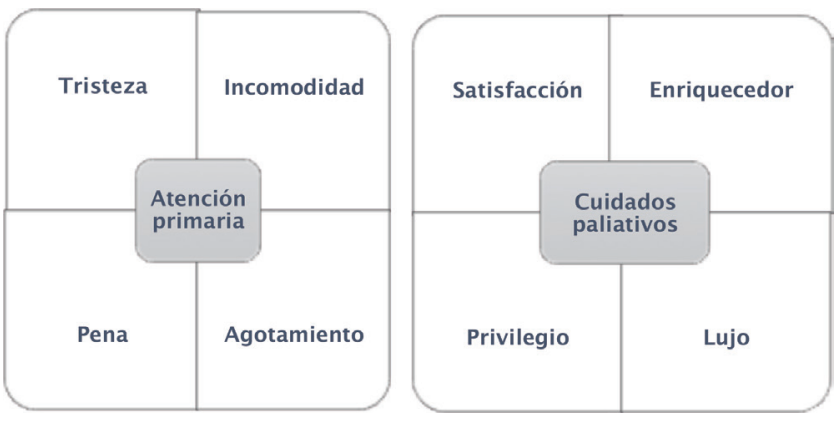

Figura 1. Perspectivas sobre las emociones en el abordaje del paciente terminal desde el punto de vista de los profesionales. Mapa conceptual.

La mayoría de los profesionales de CP se sienten privilegiados por poder atender a estos pacientes y hablar de su enfermedad. Les ayuda y aprenden escuchando a los pacientes. Lo definen como una satisfacción.

"Yo en general muy cómoda también, porque normalmente cuando hablan de la muerte con los pacientes es porque quieren hablar de ello y es muy enriquecedor. En situaciones de conspiración del silencio y pacientes no informados también reconozco un poco que incomodidad y miedo de hacer daño en esas situaciones" GFP2 (MED1).

"A mí hace que despierte, que me involucre al máximo y no pare de observarlo y ver si lo puedo llevar bien con él, ayudarlo que se encuentre mejor después de haber hablado" GFP2 (ENF1).

En los familiares, los discursos fluctuaban entre sentimientos de impotencia y soledad hasta la inexplicable fuerza que desarrollaban para ejercer el cuidado. No obstante, el sentimiento más repetido era el de sentirse generosos.

"Me sentía en una cárcel, aún tengo esa necesidad de darle más... Me sentí muy sola... Me quedaron coletillas..." FAM1.

En el caso del paciente, hablar de su enfermedad, aunque le entristecía, la mayoría de las ocasiones le tranquilizaba y le ayudaba a hablar de sus miedos, facilitándole resolver cuestiones pendientes.

"A veces lloro y no puedo parar. Me pongo muy triste y muy nerviosa... Tengo que coger aire, pero luego me relaja y me siento algo mejor, hablo con mi marido e intento comentar cosas que me gustaría resolver antes de nada" PAC1.

\section{Información y afrontamiento de la enfermedad}

Los profesionales opinaron que la verdad puede influir positivamente en el afrontamiento de la enfermedad terminal. Podría ayudar a planificar los últimos momentos, a tomar decisiones, a facilitar los aspectos emocionales y a favorecer los físicos. También creen que facilita el proceso de duelo (Figura 2).

"Saberlo implica también planificar tus últimos momentos. Saber la información te permite despedirte de la gente que quieres, conseguir una paz..." GFP2 (MED3).

Los familiares que participaron en el estudio no hablaron explícitamente sobre la enfermedad con sus allegados, aunque en ocasiones trataron sobre el cierre de cosas pendientes y se despidieron.

Para el paciente, el tener conocimiento de la información, a pesar del impacto inicial, le facilitó cerrar temas irresueltos y poder despedirse de sus allegados. También supuso participar en la toma de decisiones sobre su tratamiento.

“(...) el año que viene le tocaba la comunión a mi hija. Hablamos con el cura y la adelantamos a este año porque no creo que vaya a llegar (...) y luego el final, no quiero sufrir, no quiero sentir ahogo. Quiero que me ayudéis" PAC1.

\section{Relación según el entorno}

En esta dimensión se ha considerado la influencia de las creencias religiosas, el nivel sociocultural y el marco social (Figura 3).

Para los profesionales, que el paciente tenga fe ayuda a que esté más tranquilo, pero en ocasiones creen que para algunos creyentes la enfermedad puede asimilarse a castigo $y$, como respuesta, tengan un mayor miedo a la muerte.

En un grupo focal se habla de que es más importante el aspecto espiritual que la creencia religiosa. Algunos creyentes se alejan de su fe en momentos de rabia y enfado.

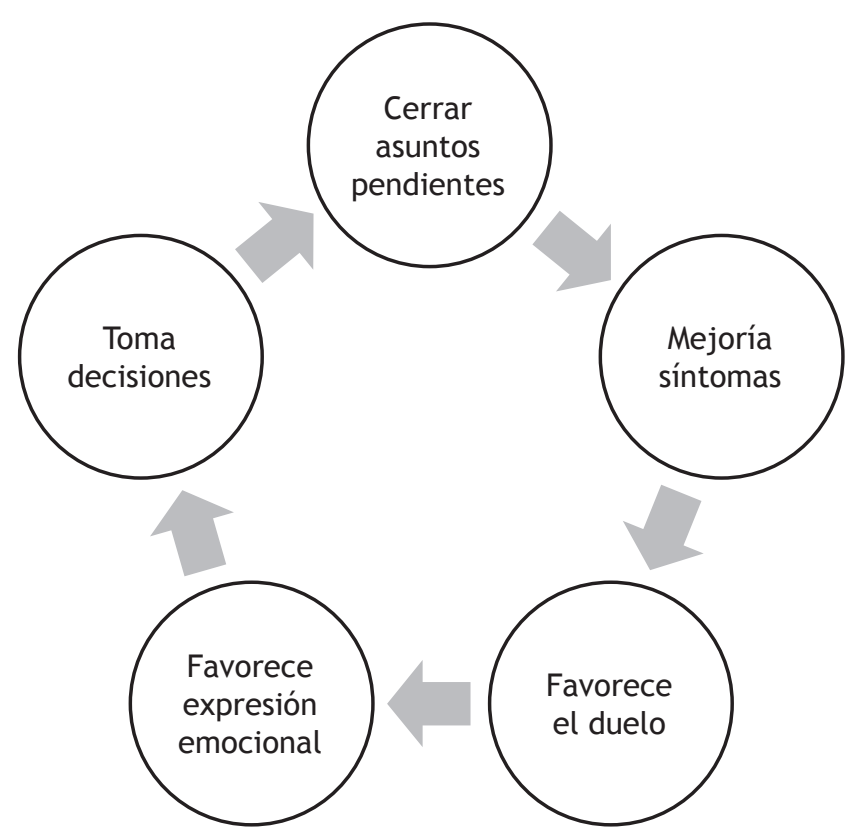

Figura 2. Beneficios de la información/conocimiento del pronóstico/diagnóstico para el afrontamiento en pacientes terminales. 


\section{ASPECTO ESPIRITUAL}
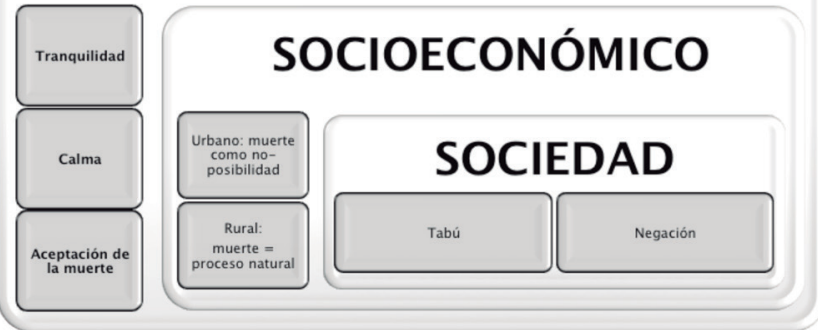

Figura 3. Factores socioculturales en la experiencia del final de la vida. Mapa conceptual.

Los familiares opinan que la religión ayuda a aceptar la muerte. En el grupo cabe destacar la opinión de un familiar con una marcada creencia religiosa argumentando que la fe ayudó a que su marido se muriera en paz y a elaborar su propio duelo.

Otro familiar es ateo, pero cree que las personas con creencias religiosas aceptan mejor la enfermedad.

\section{"Él estuvo luchando muchísimo y aceptó al Señor. Eso le} dio muchísima fuerza” GFF (FAM4).

"No creo en Dios, queremos creer en algo, creo en las voluntades, en la confianza, en el amor. (...) Mi marido no creía" GFF (FAM1).

La paciente, aunque con creencias religiosas, manifestó encontrarse en un momento de crisis de sentido.

"No sé por qué me ha tenido que tocar a mí... ¡Yo ya no creo en nada!... Soy muy joven y mi hija me necesita..." PAC1.

Los profesionales opinan que el cuidado y la visión de la muerte como natural en medio rural facilita el proceso y aceptación de la enfermedad. Se comenta que un nivel económico elevado complica en muchas ocasiones el duelo, porque les cuesta asumir lo inevitable. Parece que, desde el punto de vista de los profesionales, el tener muchos recursos económicos ofrecería una falsa sensación de poder sobre la muerte:

“El nivel socioeconómico elevado interfiere, complica las emociones, porque tienes la sensación de que puedes todo más" GFP 2 (MED1).

“Los que tienen recursos a veces tienen esa sensación de seguir. El rol del cuidador está más institucionalizado que en un ambiente más urbano" (...) "Sin embargo las que tienen menos, tienen mucha más madurez emocional y espiritual que facilita el proceso y aceptación. Aceptan mejor la vida. Ven con más naturalidad que son procesos naturales" GFP1 (MED1).

Los familiares también creen que en el medio rural la muerte se acepta mejor. El nivel socioeconómico ayuda para obtener ciertos recursos, pero el proceso de enfermedad es el mismo.
La mayoría de los profesionales creen que la muerte sigue considerándose un tabú. La sociedad sigue negándola.

"Se está muy preparado para el disfrute, la vida, el ganar, la juventud, la fuerza, pero para la decadencia, el perder facultades, no se está preparado" GFP1 (MED1).

\section{Discusión}

En esta investigación han aparecido opiniones argumentando una causa compasiva (la protección de los pacientes) para evitar el sufrimiento que supone conocer un diagnóstico negativo y un pronóstico desalentador. La relevancia del impacto emocional que experimentan los pacientes al saber de su estado es la piedra angular que justificaría el pacto de silencio. Evidentemente, la buena voluntad de quienes, en ocasiones, ocultan la información (considerado como un acto amoroso hacia sus familiares enfermos) no se puede obviar, aunque el propósito bienintencionado de no perturbarle podría conducirle a una situación de aislamiento. Dichas emociones que experimentaría el paciente sabedor de la realidad de su proceso no solo no son negativas sino que se ha descrito que conforman el proceso de adaptación a la nueva situación ${ }^{21-23}$. Se ha argumentado ${ }^{24}$ que los beneficios a corto plazo (mientras todo va bien) de ocultar la verdad o usar la ambigüedad se tornan en perjuicio porque se niega a los pacientes y a sus familias la oportunidad de reorganizarse y adaptar sus vidas hacia el logro de objetivos más realistas.

Hemos comprobado que, inicialmente, también los profesionales experimentan angustia. Algunos estudios ${ }^{25,26}$ han corroborado la influencia de las emociones en el juicio clínico de los profesionales (especialmente en el momento de diagnosticar un proceso de mal pronóstico), aunque no existe unanimidad en cuanto a las circunstancias en las que se ha de dar la información ${ }^{27}$.

Se ha visto que la aparición de una enfermedad grave produce una serie de desajustes en los miembros de la familia ${ }^{28}$. Aunque existe un acuerdo entre nuestros informantes sobre la importancia de no engañar al paciente, sí que se han omitido detalles de la enfermedad y el pronóstico, sobre todo como una decisión de los familiares. En este sentido, un estudio determinó que el $19 \%$ de los familiares no consideró la conveniencia de que su familiar conociera que tenía cáncer ${ }^{29}$.

En los estudios sobre grado de conocimiento de la enfermedad terminal se asegura que el porcentaje de enfermos que conocen la naturaleza maligna de su proceso es variable, del $21 \%$ en unos estudios ${ }^{2}$ hasta del $40-60 \%$ en otros ${ }^{10}$ o el $78 \%$ en otros ${ }^{30}$. La mayoría de las conspiraciones del silencio afectarían al pronóstico más que al desconocimiento del diagnóstico. La realidad percibida por los informantes de nuestro estudio es que el paciente sabe habitualmente bastante más de lo que se le ha dicho y que nuestros pacientes no se encuentran en una situación de desinformación absoluta. Se ha visto en este trabajo que el paciente de edad avanzada suele ser más vulnerable y quizá por eso se entienda que exista un menor conocimiento de la gravedad y menor la información recibida ${ }^{31}$.

Los profesionales de AP manifiestan mayores dificultades a la hora de comunicarse con el enfermo terminal, lo que coincide con otro estudio ${ }^{32}$ que afirma que esto responde a una falta de confianza en su capacidad profesional. Creemos 
que la adecuada formación sobre la muerte y reconocer y familiarizarse con el manejo emocional del paciente puede favorecer la empatía del profesional, reduciendo a su vez el estrés y respondiendo mejor en la atención del paciente terminal. También sería interesante significar el papel mediador del profesional, que debe responder a las necesidades del paciente más que a un estricto protocolo, en lo que a la comunicación de noticias se refiere. Hay que resaltar la importancia de que los profesionales sean capaces de crear un clima de confianza y un ambiente psicológicamente seguro para poder abordar estas cuestiones de manera efectiva. Probablemente, en la flexibilidad y adaptabilidad del personal sanitario a lo que el paciente demande esté la solución al dilema del manejo de información en estos contextos. Se ha visto ${ }^{33}$ que unas adecuadas habilidades de comunicación contribuirían a prevenir el síndrome de burnout en profesionales sanitarios. Por otra parte, los profesionales de CP experimentan emociones positivas en el manejo del paciente al final de su vida, lo que indicaría su adecuada preparación específica al respecto.

Con respecto a las creencias religiosas, consideramos, al igual que otros estudios ${ }^{34,35}$, que en las situaciones de cercanía a la muerte el aspecto espiritual debe potenciarse, ya que (no en todos los casos) genera un estado de paz y pensamiento positivo que dotaría de sentido al sufrimiento.

En el estudio se valoraron el nivel sociocultural y económico. Según otros estudios similares ${ }^{36,37}$, el conocimiento básico de los aspectos culturales que influyen en la adaptación psicosocial al cáncer facilita las interacciones entre los miembros del personal sanitario, el paciente y su familia, y permite adoptar una actitud integradora en el cuidado del paciente oncológico.

Culturalmente, la muerte y la enfermedad siguen siendo un tabú ${ }^{38}$, lo que probablemente ayude a perpetuar los miedos en la atención al paciente al final de la vida. Asimismo, la influencia de la cultura en el manejo de la información sobre un diagnóstico/pronóstico de terminalidad en el contexto español se basa en otra cosmovisión diferente al mundo anglosajón (más directo y menos eufemístico en desvelar la verdad). Se ha puesto de manifiesto ${ }^{39}$ que existe un acuerdo tácito para no desvelar inicialmente el diagnóstico de cáncer, culturalmente establecido; aunque en el fondo los pacientes se saben enfermos y advierten su gravedad (como se ha puesto de manifiesto en este trabajo), se prefiere no hablar del tema. Esta práctica de delegar en los familiares la toma de decisiones sobre la información al paciente ${ }^{13,39}$ no se entiende desde la óptica científica anglosajona que impera en occidente.

Como conclusión, podemos afirmar que el pacto de silencio tiene un marcado carácter sociocultural. No se puede responsabilizar a los profesionales o a los familiares de la situación sin antes considerar el contexto en el que se desarrolla (muerte como tabú moderno y aspectos culturales relacionados). Es preciso que los profesionales que atienden a pacientes terminales se formen en cuidados paliativos $\mathrm{y}$ en habilidades de comunicación.

\section{Limitaciones}

Los resultados de este estudio son específicos de esta muestra y contexto, y no deben ser generalizados a otras poblaciones o a contextos distintos, dado que la selección de participantes no siguió criterios estadísticos ni de representatividad (muestreo de conveniencia). Sin embargo, al tratarse de un estudio con intención exploratoria, se han identificado tendencias que puedan también aplicarse a situaciones similares en otros contextos como punto de partida.

\section{Agradecimientos}

A los familiares que han colaborado activamente en esta investigación por sus aportaciones. A la paciente cuya biografía forma parte de este trabajo por su generosidad en un momento tan delicado como era el final de su vida. A los profesionales sanitarios del Área IX del SMS y del Hospital Morales Meseguer, que han colaborado en esta investigación con sus opiniones y aportaciones.

\section{Conflicto de intereses}

Ninguno.

\section{Bibliografía}

1. Barbero J. El derecho del paciente a la información: el arte de comunicar. An Sist San Navarra 2006;29:19-27.

2. Ruíz-Benítez de Lugo MA, Coca MC. El pacto de silencio en los familiares de los pacientes oncológicos terminales. Psicooncología. 2008;5:53-70.

3. Díaz Agea JL. Aspectos psicológicos del cáncer. En: Manual de atención psicosocial. Barcelona: Monsa-Prayma; 2012. p. 199211.

4. Ramírez AMT. Evaluación del grado de información de los pacientes al ser incluidos en el programa de cuidados paliativos domiciliarios. Cuad Bioet. 2017;28:41-53.

5. Rodríguez-Castillo C. Conspiración del silencio. Rev Esp Com Sal (RECS). 2015;6:213-21.

6. Achury DM, Pinilla M. La comunicación con la familia del paciente que se encuentra al final de la vida. Enfermería universitaria. 2016;13:55-60.

7. Sánchez N, Sirgo A, Hollenstein MF, León C, Lacorte MT, Salamero M. Preferencias de comunicación y apoyo de pacientes oncológicos españoles. Adaptación del “Measure of patient's preferences”. Psicooncología. 2005;2:81-90.

8. Ruiz-Benítez de Lugo M. La comunicación y el sistema familiar en el paciente oncológico terminal. Psiquiatría Com. 2008;12(1). Disponible en https://psiquiatria.com/psicologia/la-comunicacion-y-el-sistema-familiar-en-el-paciente-oncologico-terminal/

9. Cobos FM, Almendro JE, Strempell JP, del Rosario MB. Atención a la familia. Aten Primaria. 2002;30:576-80.

10. SECPAL. Guía de Cuidados Paliativos. (2014). Disponible en: https://cmvinalo.webs.ull.es/docencia/Posgrado/8-ANCER\%20 Y\%20CUIDADOS-PALIATIVOS/guiacp.pdf

11. Reich M, Mekaoui L. Conspiracy of silence in oncology: a situation not to be overlooked. Bull Cancer. 2003;90:181-4.

12. Cobos F, Espinoza J, Portillo J, Rodríguez G. La familia en la enfermedad terminal. Med Fam. 2002;3:44-50.

13. Gómez Sancho M. Medicina Paliativa en la cultura latina. Madrid: Arán Ediciones; 1999.

14. Bermejo JC, Villacieros M, Carabias R, Sánchez E, Díaz-Albo B. Conspiración del silencio en familiares y pacientes al final de la vida ingresados en una unidad de cuidados paliativos: nivel de información y actitudes observadas. Med Paliat. 2013;20:49-59. 
15. Schettini P, Cortazzo I. Análisis de los datos cualitativos en la investigación social. Buenos Aires: Editorial de la Universidad Nacional de La Plata (EDULP); 2015.

16. Arantzamendi M, López-Dicastillo O, Robinso C, Carrasco JM. Investigación cualitativa en Cuidados Paliativos. Un recorrido por los enfoques más habituales. Med Paliat. 2017;24:219-26.

17. Schriewer K, Díaz Agea JL. Cuestiones prácticas en cuanto a la elaboración de relatos biográficos. Quaderns-e de l'Institut Català d'Antropologia. 2015;20:114-31.

18. Álvarez-Gayou Jungerson JL. Cómo hacer investigación cualitativa: Fundamentos y metodología. Barcelona: Editorial Paidós; 2003.

19. Hsieh HF, Shannon SE. Three approaches to qualitative content analysis. Qual Health Res. 2005;15:1277-88.

20. Colaizzi PF. Psychological research as the phenomenologist views it. In: Valle RS, King M (eds). Existential phenomenological alternatives for psychology. New York: Oxford University Press; 1978.

21. Worden JW, Aparicio A. El tratamiento del duelo: asesoramiento psicológico y terapia. Barcelona: Ediciones Paidós; 2003.

22. Ávila MM, de la Rubia JM. El significado psicológico de las cinco fases del duelo propuestas por Kübler-Ross mediante las redes semánticas naturales. Psicooncología. 2013;10:109-30.

23. Gómez Sancho M. Cómo dar malas noticias en medicina. Madrid: Arán Ediciones SA; 2000.

24. Fallowfield LJ, Jenkins VA, Beveridge HA. Truth may hurt but deceit hurts more: communication in palliative care. Palliat Med. 2002;16:297-303.

25. García-Caro MP, Cruz-Quintana F, Río-Valle JS, Muñoz-Vinuesa A, Montoya-Juárez R, Prados-Peña D, et al. Influencia de las emociones en el juicio clínico de los profesionales de la salud a propósito del diagnóstico de enfermedad terminal. International J Clin Health Psychol. 2010;10:57-73.

26. Dean M, Street RL. A 3-Stage model of patient-centered communication for addressing cancer patients' emotional distress. Patient Educ Couns. 2014;94:143-8.

27. Ortega-Galán AM, González-de Haro MD. La información sanitaria en el proceso de la muerte: ¿a quién corresponde? Med Paliat. 2017;24:83-8.
28. Cejudo López A, López López B, Crespo Serván P, Duarte Rodríguez M, Coronado Illescas C, de la Fuente Rodríguez C. El pacto de silencio desde la perspectiva de las personas cuidadoras de pacientes paliativos. Enferm Clínica. 2015;25:124-32.

29. Díaz-Cordobés JL, Barcia JA, Gallego-Sánchez JM, Barreto P. Conspiración de silencio y malestar emocional en pacientes diagnosticados de glioblastoma multiforme. Psicooncología. 2012;9:151-9.

30. Font-Ritort S, Martos-Gutiérrez JA, Montoro-Lorite M, MundetPons L. Calidad de la información sobre el diagnóstico al paciente oncológico terminal. Enferm Clínica. 2016;26:344-50.

31. Arribas VR, Martínez ES, Sarasola MZ, Benito IG, Redin SA, Ibáñez VT. Diagnóstico: cáncer. ¿Queremos conocer la verdad? Aten Primaria. 2004;33:368-73.

32. Mialdea MJ, Sanz J, Sanz A. Situaciones difíciles para el profesional de atención primaria en el cuidado al enfermo terminal. Psicooncología. 2009;6:53-63.

33. Leal-Costa C, Díaz-Agea JL, Tirado-González S, Rodríguez-Marín J, Van-der Hofstadt CJ. Las habilidades de comunicación como factor preventivo del síndrome de Burnout en los profesionales de la salud. An del Sist San Navarra. 2015;38:213-23.

34. Sánchez Herrera B. Bienestar espirituales de enfermos terminales y de personas aparentemente sanas. Invest y Educ Enferm. 2009;27:86-95.

35. Díaz Agea J, Leal C, Schriewer K, Echevarría P. Suffering of Traumatic Amputees in Spain: Meaning, spirituality and biomedicine. Holis Nurs Pract. 2016;30:312-21.

36. Trill MD. Influencia de la cultura en la experiencia del cáncer. Psicooncología. 2003;0:39-48.

37. Espinoza-Suárez NR, del Mar Z, Milagros C, Mejía Pérez LA. Conspiración de silencio: una barrera en la comunicación médico, paciente y familia. Rev Neuropsiquiatría. 2017;80:125-36.

38. Díaz Agea JL. Morir en un hospital. Reflexiones y comentarios a la obra de Philippe Ariés. Cultura de los cuidados. 2006;19: 10-5.

39. Olarte JMN, Guillén DG. Cultural issues and ethical dilemmas in palliative and end-of-life care in Spain. Cancer control. 2001;8:46-54. 